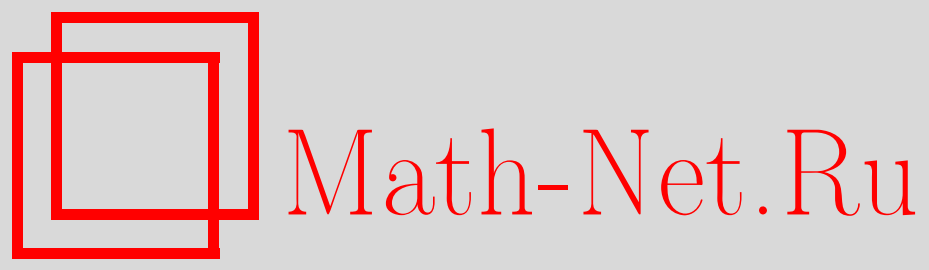

А. В. Бабин, Сохранение гомотопий и пространственно сложные решения параболических уравнений с несколькими переменными, Функи. анализ и его прил., 1996, том 30, выпуск 3, 73-76

DOI: https://doi.org/10.4213/faa539

Использование Общероссийского математического портала MathNet.Ru подразумевает, что вы прочитали и согласны с пользовательским соглашением http://www . mathnet.ru/rus/agreement

Параметры загрузки:

IP : 54.162 .27 .143

26 апреля 2023 г., 09:44:54

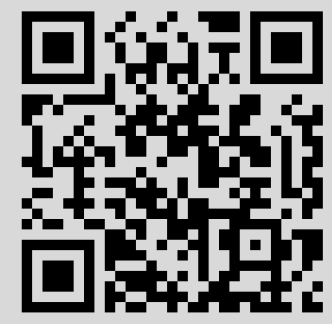




\title{
Сохранение гомотопий и пространственно сложные решения параболических уравнений с несколькими переменными
}

\author{
(C) 1996. А. В. БАБин
}

Во многих природных явлениях возникает динамика сложных пространственных форм, которые устойчиво воспроизводятся в течение длительных промежутков времени, а при наличии диссипации энергии в конце конщов застывают. Возникающие пространственные структуры часто характеризуются хаотическим, нерегулярным расположением элементов. В этой статье описывается общий и устойчивый механизм, который может объяснить возникновение и сохранение таких форм при динамике, описываемой параболическими системами. Главная идея состоит в описании сложности решения уравнения в терминах его гомотопического типа. Основной результат работы - это теорема о сохранении определенных гомотопических классов при динамике. Если энергия решений не очень велика, то эти решения принимают значения в пространстве, образованном множествами уровня нелинейного потенциала, который входит в уравнение, и имеющем нетривиальную топологическую структуру, и гомотопический класс решения определен. Если эта структура достаточно сложна, количество классов экспоненциально зависит от параметров системы и может быть очень велико. Одним из следствий является сушествование большого числа гомотопически различных решений стационарного эллиптического уравнения, образующих устойчивые компоненты. В отличие от [3-6] здесь рассмотрен случай нескольких пространственных переменных, который имеет существенные отличия от одномерного. Заметим, что классификация решений по их гомотопическому типу естественно возникает при изучении динамики отображений между многообразиями, в частности гармонических отображений (cм. $[7,8]$ и приведенную там литературу). При этом топология образа и гомотопический класс решения фиксированы с самого начала. Принципиальное отличие ситуации, рассматриваемой в данной статье, состоит в том, что здесь топология образа тривиальна и для произвольного решения гомотопический тип не определен. Только когда энергия решения диссипирует и становится достаточно малой, решение начинает чувствовать топологию, порожденную потенциалом, и возникают сохраняющиеся гомотопические классы. Некоторую аналогию можно найти с возникновением топологии в прообразе благодаря развитию сингулярностей (см. [6]).

Мы рассматриваем в ограниченной области $\Omega \subset \mathbb{R}^{n}$ параболическую систему с монотонной главной частью

$$
\partial_{t} u=\sum \partial_{i} \nabla G_{i}(\nabla u)-\nabla F(u),
$$

где $u=\left(u^{1}, \ldots, u^{d}\right) \in \mathbb{R}^{d}, d>n \geqslant 2, u=u(x, t), x \in \Omega \subset \mathbb{R}^{n}, t \geqslant 0$, $\nabla G_{i}(\nabla u)$ — градиент потенциала $G(\nabla u)$ по переменной $\partial_{i} u$, a $\nabla F(u)$ - градиент потенциала $F(u)$. Предполагается, что $F$ дважды непрерывно дифференцируема на $\mathbb{R}^{d}$ и неотрицательна, $F(u) \geqslant 0$. Потенциал $G$ трижды непрерывно дифференцируем и является неотрицательной строго выпуклой функцией. Мы 
предполагаем, что выполнены следуюшие условия:

$$
\begin{gathered}
G(0)=0, \quad a|\xi|^{p} \leqslant G(\xi) \leqslant C|\xi|^{p}+C_{1}, \\
c|\xi|^{p} \leqslant \sum G_{j}(\xi) \xi_{j} \leqslant C\left(1+|\xi|^{p}\right), \quad \xi=\left(\xi_{1}, \ldots, \xi_{d n}\right) \in \mathbb{R}^{d n}, \\
C\left(1+|\eta|^{p-2}\right)|\xi|^{2} \geqslant \sum G_{i j}(\eta) \xi_{i} \xi_{j} \geqslant 0,
\end{gathered}
$$

где $p>n, c>0, a>0, G_{j}$ являются компонентами полного градиента $G$, a $G_{i j}$ образуют матрицу его вторых производных. Функционал энергии $\mathscr{F}(u)$ имеет вид

$$
\mathscr{F}(u)=\int_{\Omega}[G(\nabla u)+F(u)] d x .
$$

Этот функционал убывает на каждом решении, $\mathscr{F}(u(T)) \leqslant \mathscr{F}(u(0))$ для любого $T \geqslant 0$, и непрерывен на $W^{1, p}(\Omega)$. Мы будем использовать пространства Соболева $W^{1, p}(\Omega), \Omega \subset \mathbb{R}^{n}$. Норма в этих пространствах задается стандартно. Мы обозначим $W^{1,2}(\mathbb{R})$ через $E$. Через $\|u\|$ мы обозначаем норму в пространстве $H$ квадратично интегрируемых функций. Через $\mathscr{S}(\Omega)$ обозначаем пространство ограниченных непрерывных на $\Omega$ функций с обычной sup-нормой. По теореме вложения Соболева $W^{1, p}(\Omega) \subset \mathscr{S}(\Omega)$ при $p>n$, т. е. $W^{1, p}(\Omega)$ состоит из непрерывных функций.

Мы рассматриваем решения системы (1), удовлетворяющие на границе (она предполагается липшицевой) граничному условию Дирихле. Пусть $E=W^{1, p}(\Omega)$ $\cap\left\{\left.u\right|_{\partial \Omega}=0\right\}$. Рассмотрим начальную задачу для (1):

$$
\left.u\right|_{t=0}=u_{0}, \quad u_{0} \in E .
$$

Теорема 1. Для любых $u_{0} \in E, T>0$ существует единственное решение $u(x, t)$ задачи $(1),(6)$, которое принадлежит $L_{\infty}([0, T], E)$. Это решение $u(t) \in E$ при фиксированном $t$ непрерьввно зависит от $u_{0} \in E$ (в слабой топологии пространства $E$ ). Справедливо неравенство

$$
\int_{0}^{T}\left\|\partial_{t} u(t)\right\|^{2} d t+\mathscr{F}(u(T)) \leqslant \mathscr{F}(u(0)), \quad T \geqslant 0 .
$$

Решение $u(t)$ непрерывно зависит от $t$ в $\mathscr{S}(\mathbb{R}), u(x, t)$ является непрерывной функиией $(x, t)$.

Доказательство стандартно; см., например, [1]. Очевидно, что из (7) следует убывание $\mathscr{F}(u(t))$.

Введем обозначения, которые нам нужны для определения сохраняющихся гомотопических классов решений системы (1).

Пусть $\omega_{n}$ - единичная сфера в $\mathbb{R}^{n}$ и $\omega^{\prime} \subset \omega_{n}$ - подмножество единичных векторов с ненулевой мерой. Пусть $y \in \Omega_{0} \subset \mathbb{R}^{n}$, где $\Omega_{0}$ - открытое подмножество в $\mathbb{R}^{n}$. Для $\nu \in \omega^{\prime}$ проведем луч с началом в $y$ параллельно $\nu$. Обозначим через $r_{0}(\nu)$ длину максимального интервала $I$ на этом луче, начинающегося в $y$ и целиком лежащего в $\Omega_{0}$; мы предполагаем, что $0<r_{0}(\nu)<\infty$. Обозначим через $\Omega^{\prime} \subset \Omega_{0}$ объединение внутренних точек из $I$ по всем $I$, и пусть

$$
\sigma\left(\omega^{\prime}, y, \Omega_{0}, \alpha\right)=\int_{\omega^{\prime}} r_{0}(\nu)^{\alpha} d \nu
$$


Пусть $\left|\omega^{\prime}\right|$ и $\left|\Omega^{\prime}\right|$ суть $(n-1)$-мерный и $n$-мерный объемы множеств $\omega^{\prime}$ и $\Omega^{\prime}$ соответственно.

Лемма 1. Пусть $0<\alpha \leqslant n$. Тогда $\sigma\left(\omega^{\prime}, y, \Omega_{0}, \alpha\right) \leqslant\left|\omega^{\prime}\right|^{1-\alpha / n} n^{\alpha}\left|\Omega_{0}^{\prime}\right|^{\alpha}$.

Если $P$ - множество из $\mathbb{R}^{d}$, обозначаем его дополнение через $P^{\prime}, P^{\prime}=$ $\mathbb{R}^{d} \backslash P$. При $0 \in P^{\prime}$ мы обозначаем через $\pi\left(\Omega, P^{\prime}, 0\right)$ множество классов гомотопных отображений $u: \Omega \rightarrow P^{\prime}$ области $\Omega$ с отмеченной точкой $*=\partial \Omega$ в $P^{\prime}$, $u(\partial \Omega)=0$ (см., например, [2]). Положим при $n<q<p$

$$
J(\omega)=\int_{s_{0}}^{s_{1}}(B F(w(s)))^{1 / q-1 / p} d s, \quad B=\frac{p(p a / q)^{q /(p-q)}}{p-q} .
$$

Якобиево расстояние $D(b, P)$ от точки $b$ до множества $P$ равно нижней грани $J(\omega)$ по всем кривым $w$, соединяющим $b$ и $P$. Положим,

$$
D(\Gamma, P)=\inf \{D(b, P), b \in \Gamma\} .
$$

Лемма 2. Пусть $y \in \Omega_{0}$, где область $\Omega_{0}$ лежит в $\Omega$. Пусть $u \in W^{1, p}(\Omega)$ $\subset \mathscr{S}(\Omega)$, причем $u(x) \in \Gamma \subset \mathbb{R}^{d}$, когда $x \in \partial \Omega_{0}$. Пусть $\omega^{\prime} \subset \omega_{n}$,

$$
\left|\omega^{\prime}\right|^{q} D(\Gamma, P)^{q}>\mathscr{F}(u) \sigma\left(\omega^{\prime}, y, \Omega_{0}, \alpha\right)^{q-1} \alpha^{1-q},
$$

где $\alpha=(q-n) /(q-1)$. Тогда суиествует $\eta>0$, maкое, ито $\operatorname{dist}(u(y), P) \geqslant \eta$.

ЗАмЕЧАНИЕ. Если $F(u) \geqslant M$ в $r$-окрестности множества $P$ и $\Gamma=0$ лежит вне $r$-окрестности, то

$$
D(0, P) \geqslant B_{1} a^{1 / p} r M^{(p-q) / q p},
$$

где $B_{1}$ зависит от $p, q$, т.е. $D(0, P)$ велико при большом $M$. Условие $(8)$ выполнено, если

$$
n^{n-q}\left|\Omega_{0}^{\prime}\right|^{1-q / n} \alpha^{q-1}\left|\omega^{\prime}\right|^{q / 2}\left(B_{1} a^{1 / p} r M^{(p-q) / q p}\right)^{q}>\mathscr{F}(u) .
$$

Следующая теорема, являющаяся следствием леммы 2, позволяет определить гомотопические классы $h(u(t))$ для решений $(1),(6)$ с начальными данными $u_{0}$, если $\mathscr{F}\left(u_{0}\right)$ не слишком велико.

Теорема 2. Пусть $u \in E$. Пусть при всех у выполнено (8), аде $\Gamma=0, a$ $\omega^{\prime}=\omega_{n}$. Тогда суиествует $\eta>0$, такое, что $\operatorname{dist}(u(x), P) \geqslant \eta$ для всех $x$. Гомотопический класс $h(u) \in \pi\left(\Omega, P^{\prime}, 0\right)$ однозначно определен. Если $u=$ $u(t)=u(x, t)$ являетсл решением системь $(1)$, то $h(u(t)) \in \pi\left(X, P^{\prime}, 0\right)$ не зависит от $t$ непрерьвно зависит от $u(0)=u_{0} \in E$. Cуществует решение $Z(x)$ стационарного уравнения

$$
0=\sum \partial_{i} \nabla G_{i}(\nabla Z)-\nabla F(Z)
$$

принадлежащее тому же гомотопическому классу, что $и(0)$.

ПримеР 1. Мы рассматриваем $n=2, d=3$. В качестве $\Omega$ берем круг радиуса $L$ с центром в нуле, а в качестве $P$ берем две точки, $P=P_{1} \cup P_{2}$. В этом случае $\pi\left(\Omega, P^{\prime}, 0\right)$ совпадает со второй гомотопической группой $\pi_{2}\left(P^{\prime}, 0\right)-$ это абелева группа с двумя образующими.

Берем $P_{1}=(-R, 0, R), P_{2}=(R, 0, R), r<R / 2$. Предполагается, что $F$ велика вблизи этих точек, $F(u) \geqslant M$, когда $\operatorname{dist}(u, P) \leqslant r$. На большом расстоянии от $P F$ меньше: $F(u) \leqslant \mu$, когда $\operatorname{dist}(u, P) \geqslant R / 2, \mu<M$. Очевидно, 
для любого $h \in \pi\left(\Omega, P^{\prime}, 0\right)$ найдется $u \in h \cap E$, такая, что $\operatorname{dist}(u(x), P) \geqslant R / 2$ для всех $x \in \Omega$. Изменяя $F$ в $r$-окрестности множества $P$, мы не влияем на $\mathscr{F}(u)$. Поэтому, взяв большое $M$, мы добьемся того, что $D(0, P)$ становится большим, и условие $(8)$ на $\mathscr{F}(u)$ теоремы 2 выполнено.

Пример 2. Мы берем $n=2, d=3$. Пусть $\Omega$ - полоса $\left|x_{2}\right|<L$ в $\mathbb{R}^{2}$. Накладываем условие $2 L_{0}$-периодичности по $x_{1}, u\left(x_{1}+2 L_{0}, x_{2}\right)=u\left(x_{1}, x_{2}\right)$, и условие Дирихле по $x_{2}, u\left(x_{1}, \pm L\right)=0$. Через $E$ обозначаем пространство функций из $W^{1, p}\left(\Omega \cap\left\{\left|x_{1}\right|<4 L_{0}\right\}\right)$, удовлетворяющих указанным граничным условиям. Утверждения и доказательства теорем 1 и 2 сохраняются в этом случае без изменений $\left(\Omega=\left\{-L_{0}, L_{0}\right\} \times\{-L, L\}\right)$.

Пусть $P$ состоит из двух окружностей $P_{1}, P_{2}$ радиуса $R_{1}=R+1$ в плоскостях $u_{3}= \pm R$, задаваемых уравнением $\left|u_{1}\right|^{2}+\left|u_{2}\right|^{2}=R_{1}^{2}$. Предполагается, что $F(u) \geqslant M$, когда $\operatorname{dist}(u, P) \leqslant r$, и $F(u) \leqslant \mu$, когда $\operatorname{dist}(u, P) \geqslant R / 2, \mu<M$. Пусть также $C_{1}=0$ в $(2)$.

Отождествив поточечно отрезки $\left(0, x_{2}\right)$ и $\left(2 L_{0}, x_{2}\right),-L \leqslant x_{2} \leqslant L$, получаем цилиндр; отождествив верхнюю и нижнюю окружности $x_{2}= \pm L$ в одну отмеченную точку $*$, получаем пространство $X$, гомеоморфное двумерной сфере с двумя отождествленными точками. Вследствие граничных условий и непрерывности функций из $E$ эти функции непрерывны на $X$.

Множество классов $\pi\left(X, P^{\prime}, 0\right)$ гораздо богаче, чем в предыдущем примере. Явно строя начальные данные $u(x, 0)$, принадлежащие заданному гомотопическому классу, можно показать, что имеется не менее

$$
N=\exp \left(\delta(r / R)(M / \mu)^{1 / q-1 / p}\right), \quad \delta>0,
$$

различных сохраняющихся гомотопических классов $h(u) \in \pi\left(X, P^{\prime}, 0\right)$ решений системы (1) с описанными $G$ и $F$; при этом решения $u(t)$ таковы, что $\mathscr{F}(u(t))$ удовлетворяет условию (8) и $h(u(t)) \in \pi\left(X, P^{\prime}, 0\right)$. В частности, есть не менее $N$ решений стационарного уравнения $(9)$.

\section{ЛитературА}

1. Бабин A. В., Вишик M. В. Аттракторы эволюционных уравнений. Наука, М. (1989). 2. Фукс Д. Б., Фоменко А. Т., Гутенмахер В. Л. Гомотопическая топология. Изд-во Моск. унив., М. (1969). 3. Afraimovich V., Babin A., Chow S.-N. Spatial Chaotic Structure of Attractors of Reaction-Diffusion Systems. Preprint CDSNS 94-185 (1994). 4. Babin A. V. Homotopy stable spatially chaotic waves on an infinite interval, to be published in Proc. of Symposium on Structure and Dynamics of Nonlinear Waves in Fluids, Hannover (1994). 5. Babin A., Bunimovich L. Dynamics of stable chaotic waves generated by hyperbolic PDE, to appear. 6. Bethuel F., Brezis H., Helen F. Ginzburg-Landau Vortices. Birkhauser (1994). 7. Brezis $H . \quad S^{k}$-valued maps with singularities. Lect. Notes in Math., Vol. 1365, Springer-Verlag (1989), pp. 1-30. 8. Hamilton $R$. Harmonic maps of manifolds with boundary. Lect. Notes in Math., Vol. 471, Springer-Verlag (1975). 